Supporting information for

\title{
Fragment Mass Spectrum Prediction Facilitates Site Localization of Phosphorylation
}

\author{
Yi Yang ${ }^{\dagger}$, Peter Horvatovich ${ }^{\dagger} * *$ and Liang Qiao $^{\dagger, *}$
}

$\dagger$. Department of Chemistry and Shanghai Stomatological Hospital, Fudan University, Handan Road 220, Shanghai 200000, China

†. Department of Pharmacy, University of Groningen, Antonius Deusinglaan 1, 9700

AD Groningen, The Netherlands

*: Correspondence should be addressed to Prof. Liang Qiao (liang_qiao@fudan.edu.cn)

or Prof. Peter Horvatovich (p.1.horvatovich@ rug.nl)

\section{Table of Content}

Text S1. Statistics of water neutral loss peaks.

Text S2. Similarities of predicted spectra to the experimental spectra.

Text S3. Benchmarks of neutral loss peak generation and budding strategies. S-6

Text S4. Collision energy (CE) calibration for the HeLa data set.

Text S5. Analysis of doubly phosphorylated peptides from HeLa cells.

Table S1. LC-MS/MS data sets used in this study.

Table S2. Probability thresholds at 1\% false localization rate (FLR).

Figure S1. Optimization of CE value for Prosit and pDeep2, as well as phosphoRS parameters on the Syn-OF data set. 
Figure S3. Dot product between the extracted peaks from the experimental spectra and the predicted spectra.

Figure S4. DeltaDP results of matching the extracted peaks from the experimental spectra and the predicted spectra with different candidate phosphorylation sites. S-14 Figure S5. Phosphorylation site localization using MS/MS spectra predicted by pDeep2 with/without buddings.

Figure S6. Phosphorylation site localization on Syn-OF, Syn-QE and Syn-TOF using different methods with neutral loss and budding.

Figure S7. Localization results on the Syn-QE data set using different methods. S-17 Figure S8. Localization results on the Syn-TOF data set using different methods.S-18 Figure S9. Distributions of distance between the mislocated phosphorylation sites and the correct sites in Syn-OF using different methods.

Figure S10. Optimization of CE value for pDeep2 on the HeLa data set.

Figure S11. Phosphorylation site localization of doubly phosphorylated peptides from HeLa cells.

Data Set S1. Phosphorylation site localization results on Syn-OF by Ascore and phosphoRS, as well as spectral matching using MS/MS spectra predicted by $\mathrm{MS}^{2} \mathrm{PIP}$, Prosit, DeepMS2, and pDeep2. (Separated XLSX)

Data Set S2. Phosphorylation site localization results on Syn-OF using spectral matching by DeepMS2 with different neutral loss ratios. (Separated XLSX)

Data Set S3. Phosphorylation site localization results on Syn-OF using spectral matching by DeepMS2 with different budding factors. (Separated XLSX) 
Data Set S4. Phosphorylation site localization results on Syn-OF using spectral matching by DeepMS2, MS 2 PIP and Prosit with neutral loss generation and budding.

(Separated XLSX)

Data Set S5. Phosphorylation site localization results on Syn-QE by Ascore and phosphoRS, as well as spectral matching using MS/MS spectra predicted by $\mathrm{MS}^{2} \mathrm{PIP}$, Prosit, DeepMS2, and pDeep2.

(Separated XLSX)

Data Set S6. Phosphorylation site localization results on Syn-TOF by Ascore and phosphoRS, as well as spectral matching using MS/MS spectra predicted by $\mathrm{MS}^{2} \mathrm{PIP}$, Prosit, DeepMS2, and pDeep2.

(Separated XLSX)

Data Set S7. Phosphorylation site localization results on Syn-OF, Syn-QE and SynTOF by phosphoRS combined with spectral matching.

(Separated XLSX)

Data Set S8. Phosphorylation site localization results for singly phosphorylated peptides from the HeLa data set by phosphoRS, as well as phosphoRS combined with spectral matching.

(Separated XLSX)

Data Set S9. Phosphorylation site localization results for doubly phosphorylated peptides from the HeLa data set by phosphoRS, as well as phosphoRS combined with spectral matching.

(Separated XLSX) 
Text S1. Statistics of water neutral loss peaks.

We performed statistics of water neutral loss $(-18)$ peaks from 897,477 spectra of $2+$ non-phosphorylated peptide precursors and 760,920 spectra of 3+ non-phosphorylated peptide precursors in the MassIVE-KB Human HCD Spectral Library (https://massive.ucsd.edu/ProteoSAFe/static/massive-kb-libraries.jsp). Peak annotation was performed using spectrast2tsv.py (https://github.com/msproteomicstools/msproteomicstools). The numbers of water neutral loss peaks were $\sim 25 \%$, in median, of those of peaks without neutral loss, and the intensity sums of water neutral loss peaks were $<10 \%$, in median, of those of peaks without neutral loss (Supporting Information Figure S2).

Water neutral loss are frequently observed, and might be misrecognized as phosphoric acid neutral loss of phosphate-containing fragments (+80-98), increasing false localization in probability-based localization methods. In this case, phosphoric acid neutral loss peaks should not be used as site-specific peaks. However, spectral matching does not count any site-specific peaks, but takes into account the whole fragment intensity pattern of the spectra. Adding phosphoric acid neutral loss peaks makes the predicted spectra more similar to the experimental spectra (Figure S3), and improves localization performance. 
Text S2. Comparison of similarities of predicted spectra to the experimental spectra. We compared the similarities of predicted spectra to the experimental spectra using a phosphopeptide spectral library containing $>100 \mathrm{k}$ unique localized phosphopeptides from Phosphopedia (https://phosphopedia.gs.washington.edu/PhosphoproteomicsAssay/sections/elib.xhtm 1). From the library we collected 26,997 experimental spectra of $2+$ singly phosphorylated peptide precursors, and 39,428 of $3+$ singly phosphorylated peptide precursors (one spectrum per precursor). Peaks of $\mathrm{b} / \mathrm{y}$ ions with/without phosphoric acid neutral loss (NL) were annotated and extracted using spectrast2tsv.py (https://github.com/msproteomicstools/msproteomicstools). Dot product (DP) was calculated between the extracted peaks from the experimental spectra and the predicted spectra for each phosphopeptide (Supporting Information Figure S3). We also calculated DP between the extracted peaks from the experimental spectra and the predicted spectra of phosphopeptides with the same stripped sequence but different phosphorylation sites. For each experimental spectrum, deltaDP was calculated between the top match (correct or incorrect) and the second match (Supporting Information Figure S4). Ambiguous results (deltaDP $=0)$ were excluded.

DPs of spectra predicted by DeepMS2, MS 2 PIP and Prosit after adding NL peaks ( $p \%$ $=50 \%$ ) were higher than those without NL, and were close to those predicted by pDeep2. After budding $(b \%=10 \%)$, DPs decreased due to spectral distortion. However, deltaDP ratios of correct/incorrect matches after budding were higher than those without budding, indicating that budding benefits separation between correct and incorrect matches. 
Text S3. Benchmarks of neutral loss peak generation and budding strategies.

From Syn-OF, 1012, 958, 1004 and 812 PSMs were correctly localized at 1\% FLR using spectra predicted by DeepMS2, MS ${ }^{2} \mathrm{PIP}$, Prosit and pDeep2 with $50 \%$ of S/T neutral loss and 10\% budding, respectively (Supporting Information Figure S6A and Data Set S4), more than those by phosphoRS (730 PSMs) and pSite (624 PSMs).

Benchmarks were further performed on Syn-QE and Syn-TOF (Supporting Information Table S1). The CE parameter was optimized for Prosit and pDeep2, while the neutral loss option was tested for phosphoRS (Supporting Information Figure S7 and S8, and Data Set S5 and S6). For DeepMS2, MS ${ }^{2}$ PIP and Prosit, the $p \%$ and $b \%$ parameters were the same as those used on Syn-OF. From HCD spectra of Syn-QE acquired on a Q Exactive, 1070, 1018 and 960 PSMs were correctly localized at 1\% FLR by MS ${ }^{2}$ PIP, DeepMS2 and Prosit, respectively, more than those by phosphoRS (702 PSMs), less than those by pSite (1073 PSMs), and pDeep2 correctly localized the most PSMs (1370 PSMs) as shown in Figure S6B. From CID spectra of Syn-TOF acquired on a TripleTOF, pDeep2 and MS²PIP correctly localized 2293 and 2073 PSMs at 1\% FLR, respectively. Prosit and DeepMS2 correctly localized 1543 and 1791 PSMs at 1\% FLR, respectively, more than those of phosphoRS (1393 PSMs) and Ascore (734 PSMs) as indicated in Figure S6C. Notably, the FLR curves of MS²PIP, DeepMS2 and Prosit were converging with that of pDeep2 and away from that of phosphoRS towards $5 \%$ FLR. Indeed, the global FLRs of all the PSMs reported by MS ${ }^{2}$ PIP, DeepMS2 and Prosit were $<5 \%$. 
Text S4. Collision energy (CE) calibration for the HeLa data set.

Since the actual phosphorylation sites of peptides in the HeLa data set of a real sample were unknown, we were not able to perform CE calibration as what we did with SynOF. Therefore, CE was optimized using the spectra of non-phosphorylated peptides in the HeLa data set. For Prosit, the raw data were searched without phosphorylation using MaxQuant, and the raw and result files were submitted to the Prosit web service. The optimized CE was 37.

For pDeep2, the mzML files were searched using Comet, and the results were filtered by $1 \%$ FDR using PeptideProphet at peptide level. A spectral library was built from the search results using SpectraST, and b/y ions were extracted using spectrast2tsv.py script. DP was computed between the intensities of the extracted b/y ions from the experimental MS/MS spectra and those predicted by pDeep2 with different CEs (Figure S10). The optimized CE was 36. 
Text S5. Analysis of doubly phosphorylated peptides from a data set acquired from HeLa cells.

A LC-MS/MS dataset from HeLa cells (PXD000474) includes 830 PSMs of 323 peptide sequences that satisfy the criteria described below: (i) with two phosphorylation motifs at serine, threonine or tyrosine; (ii) with $\geq 3$ candidate phosphorylation sites (serine, threonine and tyrosine); (iii) with $2+$ or $3+$ charge states. These PSMs were used to evaluate the localization performance by our methods.

At a probability threshold of 99.5\%, phosphoRS localized 378 PSMs of 174 phosphopeptides (Figure S11A and Support Information Data Set S9). Using DPphosphoRS with in silico spectral libraries predicted by MS²PIP, DeepMS2 and Prosit with neutral loss and budding optimization, 529, 537 and 530 PSMs of 227, 229 and 225 phosphopeptides were localized, respectively. Using in silico spectral libraries predicted by pDeep2, 539 PSMs of 230 phosphopeptides were localized. For Prosit and pDeep2, CE value was set to 37 and 36 , respectively.

For the PSMs localized by phosphoRS alone, DP-phosphoRS mostly (>97\%) reported the same results, while only few $(\sim 2 \%)$ were missed. At the phosphopeptide level, DP-phosphoRS localized $>97 \%$ phosphopeptides of those localized by phosphoRS alone and reported $>30 \%$ new phosphopeptides. In addition, DPphosphoRS showed higher $(>65 \%)$ overlaps of phosphopeptide localization between two technical replicates than phosphoRS alone (Figure S11B).

The results indicate that our method is promising in site localization of multiply phosphorylated peptides, although further optimization and benchmarking should be performed using large data sets of annotated phosphopeptides with ground truth, i.e. using high purity synthetic phosphorylated peptides. 
Table S1. LC-MS/MS data sets used in this study.

\begin{tabular}{|l|l|l|l|}
\hline Code & Instrument & Description & Accession \\
\hline $\begin{array}{l}\text { Syn- } \\
\text { OF }\end{array}$ & Orbitrap Fusion & $\begin{array}{l}\text { HCD MS/MS spectra of synthetic phosphopeptides. } \\
\text { pS: 713 PSMs, 87 phosphopeptides; } \\
\text { pT: 174 PSMs, 20 phosphopeptides; } \\
\text { pY: 336 PSMs, 33 phosphopeptides; } \\
\text { Total: 1223 PSMs, 140 phosphopeptides, 106 } \\
\text { sequences. }\end{array}$ & PXD007058 \\
\hline $\begin{array}{l}\text { Syn- } \\
\text { QE }\end{array}$ & Q Exactive & $\begin{array}{l}\text { HCD MS/MS spectra of synthetic phosphopeptides. } \\
\text { pS: 1214 PSMs, 14 phosphopeptides; } \\
\text { pT: 276 PSMs, 3 phosphopeptides; } \\
\text { pY: 147 PSMs, 3 phosphopeptides; } \\
\text { Total: 1637 PSMs, 20 phosphopeptides, 11 sequences. }\end{array}$ & PXD000474 \\
\hline $\begin{array}{l}\text { Syn- } \\
\text { TOF }\end{array}$ & TripleTOF 6600 & $\begin{array}{l}\text { CID MS/MS spectra of synthetic phosphopeptides. } \\
\text { pS: 2292 PSMs, 34 phosphopeptides; } \\
\text { pT: 226 PSMs, 6 phosphopeptides; } \\
\text { pY: 209 PSMs, 1 phosphopeptides; } \\
\text { Total: 2727 PSMs, 41 phosphopeptides, 15 sequences. }\end{array}$ & PXD012433 \\
\hline HeLa & $\begin{array}{l}\text { LTQ Orbitrap } \\
\text { Velos }\end{array}$ & $\begin{array}{l}\text { 4786 PSMs, 1677 sequences for singly } \\
\text { phosphorylated peptides, and 830 PSMs, 323 } \\
\text { sequences for doubly phosphorylated peptides. }\end{array}$ & PXD000474 \\
\hline
\end{tabular}


Table S2. Combined spectral matching and phosphoRS probability thresholds corresponding to $1 \%$ false localization rate (FLR).

\begin{tabular}{|l|l|l|l|}
\hline & Syn-OF & Syn-QE & Syn-TOF \\
\hline MS2PIP-phosphoRS & 99.31 & 98.28 & 99.70 \\
\hline Prosit-phosphoRS & 99.58 & 97.68 & 99.74 \\
\hline DeepMS2-phosphoRS & 99.57 & 99.07 & 99.74 \\
\hline pDeep2-phosphoRS & 97.37 & 95.14 & 99.22 \\
\hline phosphoRS alone & 99.58 & 99.69 & 99.31 \\
\hline
\end{tabular}



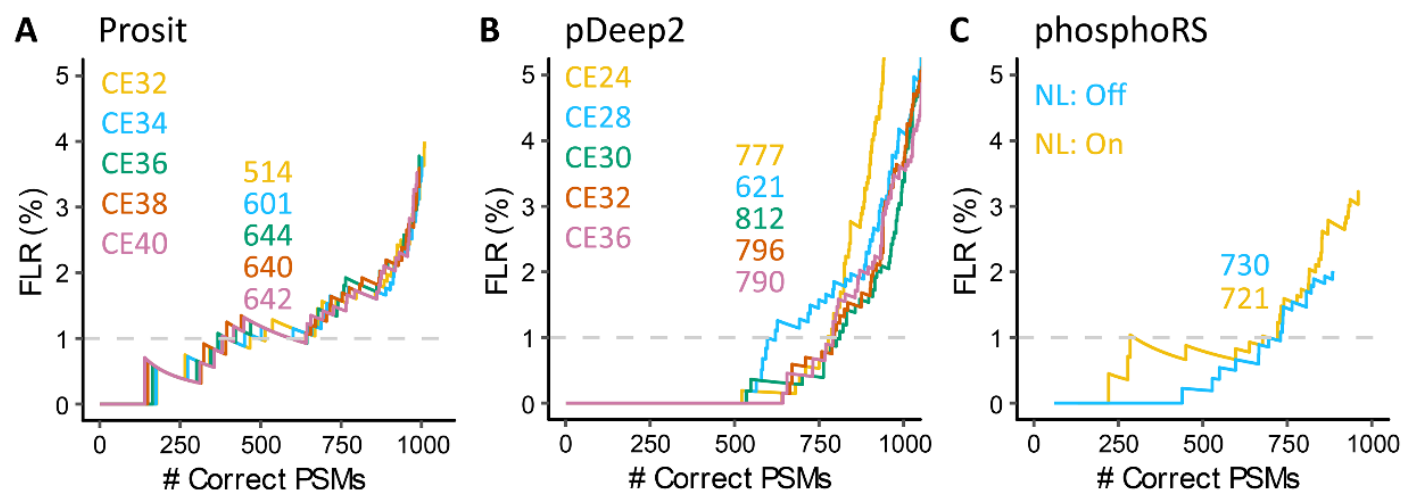

Figure S1. Phosphorylation site localization on Syn-OF using different methods. (A) Plots showing PSM level FLR against the number of correctly localized PSMs by spectral matching using MS/MS spectra predicted by Prosit with different collision energy (CE) values. (B) Results of spectral matching using MS/MS spectra predicted by pDeep2 with different CE values. (C) Results of phosphoRS with the neutral loss (NL) option enabled or turned off. The numbers of correctly localized PSMs at 1\% FLR are indicated. Localization results of PSMs are sorted by the deltaDP scores or phosphoRS probabilities in descending order. 
A Charge $2+(897,477$ spectra $)$
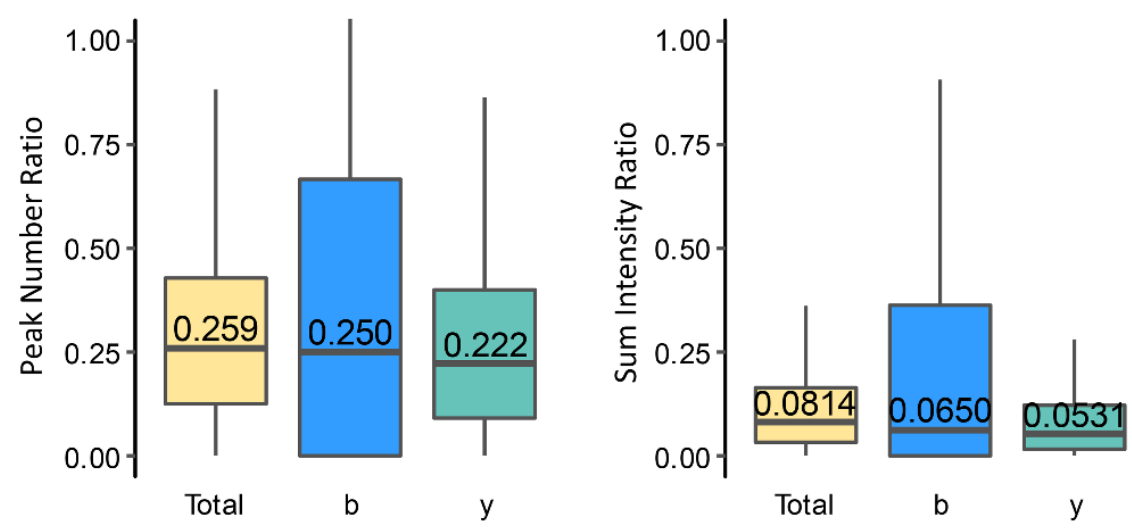

B Charge $3+(760,920$ spectra $)$
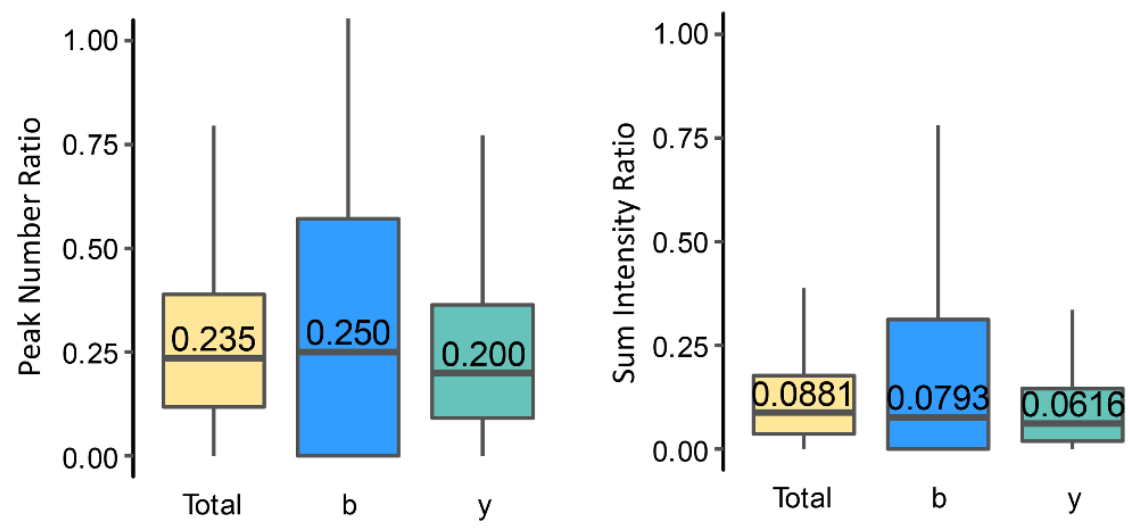

Figure S2. Statistics of water neutral loss peaks from spectra of $2+(A)$ and $3+(B)$ nonphosphorylated peptide precursors. Peak number ratio: number of water neutral loss peaks / number of peaks without neutral loss; Sum intensity ratio: intensity sum of water neutral loss peaks / intensity sum of peaks without neutral loss. The medians are indicated. The boxes show interquartile ranges (IQR); the upper/lower whiskers show the largest/smallest values no further than $1.5 *$ IQR from the quantiles; no outliers are shown. 


\section{A DeepMS2}

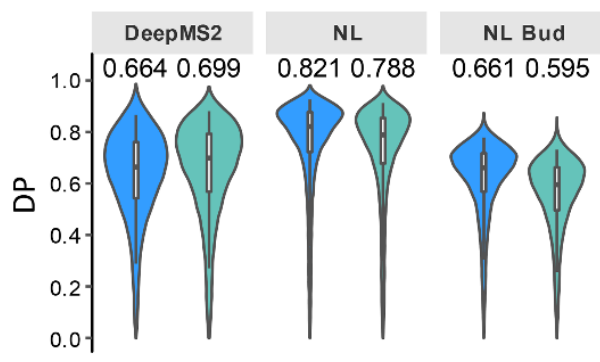

\section{Prosit}

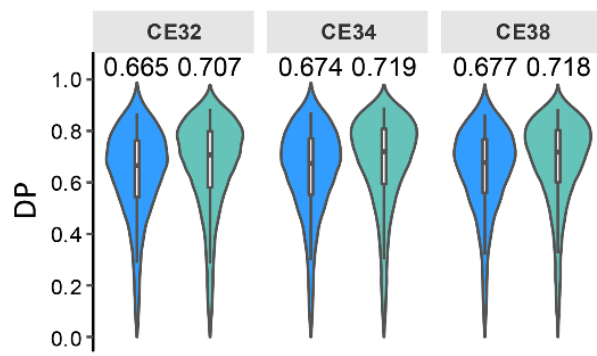

\section{B MS'PIP $^{2}$}
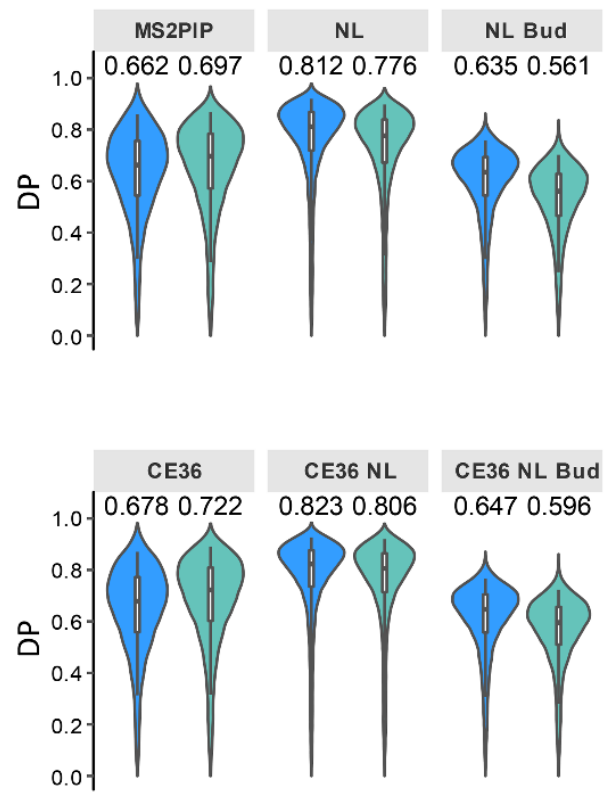

\section{D pDeep2}

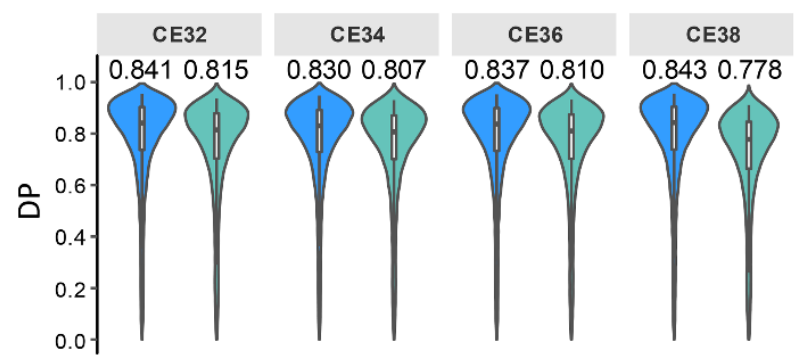

$\square 2+$

$\square 3+$

Figure S3. Dot product (DP) between the extracted peaks from the experimental spectra and the predicted spectra. NL: after adding phosphoric acid neutral loss peaks $(p \%=$ $50 \%)$; Bud: after budding $(b \%=10 \%)$; CE: collision energy. The medians are indicated. The boxes show interquartile ranges (IQR); the whiskers show the $95 \%$ percentiles; no outliers are shown. 
A DeepMS2

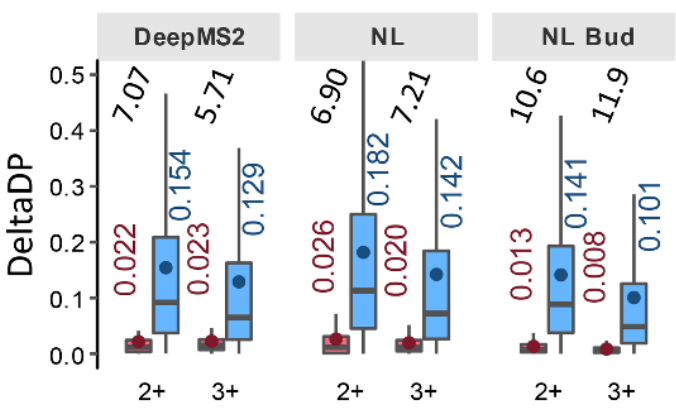

C Prosit

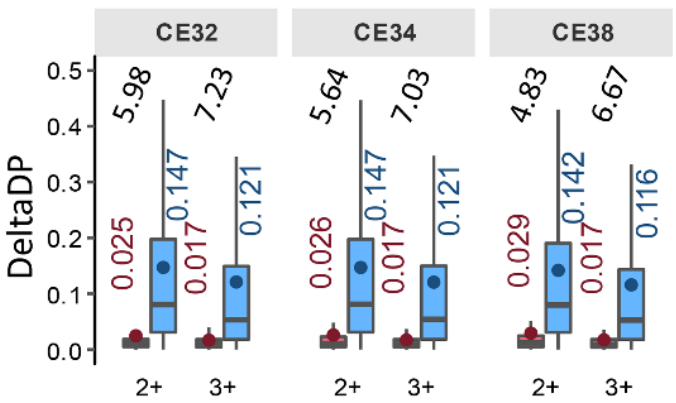

\section{B MS $^{2}$ PIP}

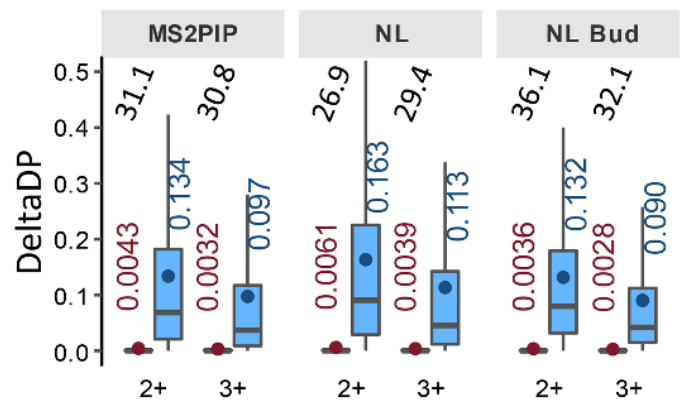

\section{D pDeep2}
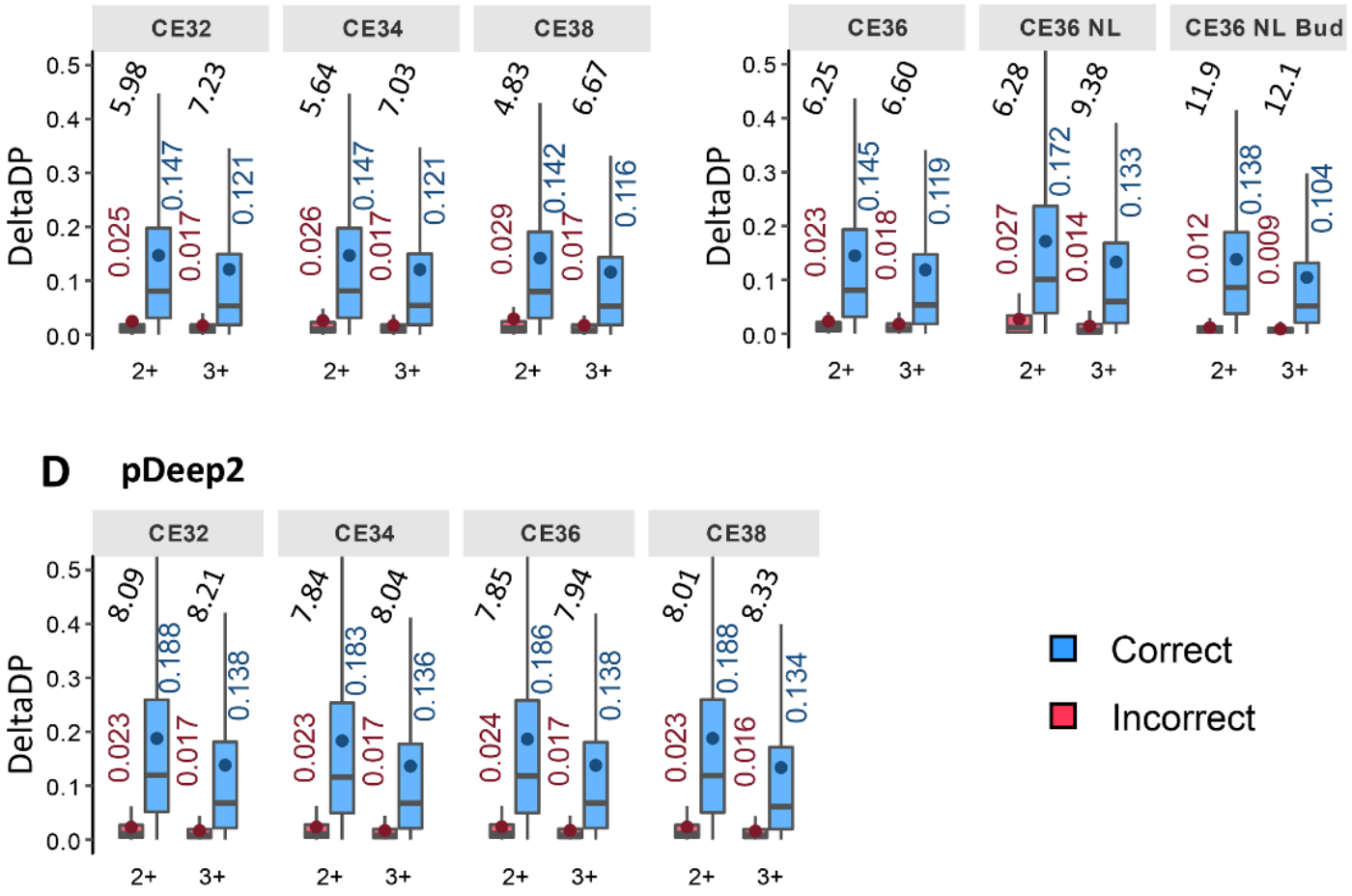

$\square$ Correct

$\square$ Incorrect

Figure S4. DeltaDP results of matching the extracted peaks from experimental spectra and predicted spectra with different candidate phosphorylation sites. NL: after adding phosphoric acid neutral loss peaks $(p \%=50 \%)$; Bud: after budding $(b \%=10 \%)$; CE: collision energy; Correct: the top match is the predicted spectrum of the same phosphopeptide as the experimental spectrum; Incorrect: the top match is the predicted spectrum of different phosphorylation sites from the experimental spectrum. The mean deltaDPs (in red or blue characters) and their ratios (correct/incorrect, in black characters) are indicated. The boxes show medians and interquartile ranges (IQR); the upper/lower whiskers show the largest/smallest values no further than $1.5 *$ IQR from the quantiles; no outliers are shown. 
A

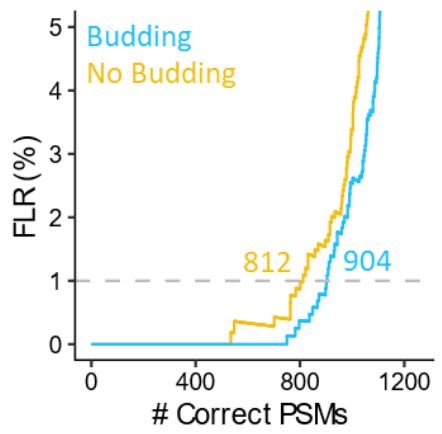

B

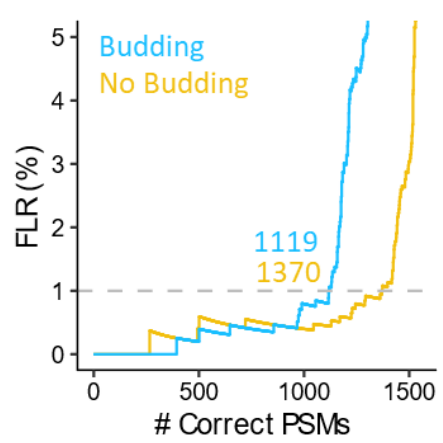

C

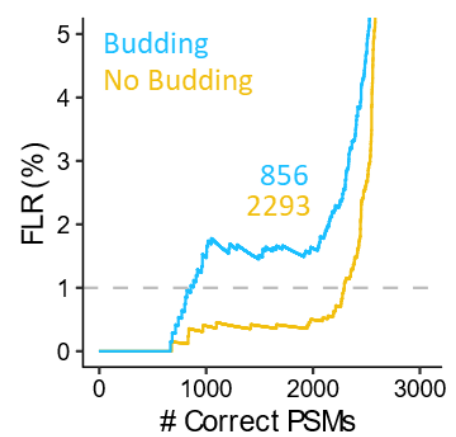

Figure S5. Phosphorylation site localization on the data sets Syn-OF (A), Syn-QE (B) and Syn-TOF (C) using MS/MS spectra predicted by pDeep2 with/without $10 \%$ buddings. Buddings led to better results for Syn-OF and worse results for Data sets SynQE and Syn-TOF. 

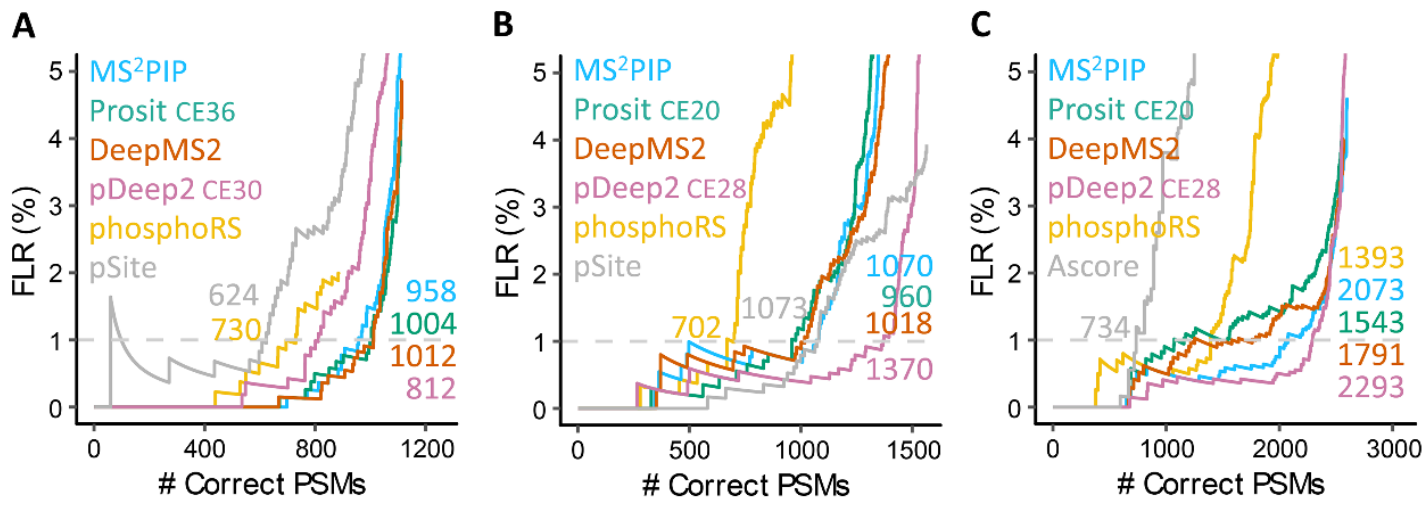

Figure S6. Phosphorylation site localization on the data sets Syn-OF, Syn-QE and SynTOF using different methods. (A) Plots of PSM level FLR against the number of correctly localized PSMs from Syn-OF by pSite and phosphoRS, as well as spectral matching using MS/MS spectra predicted by $\mathrm{MS}^{2} \mathrm{PIP}$, Prosit and DeepMS2 with neutral loss peak generation (50\% S/T) and budding (10\%), and by pDeep2. (B) Localization results from Syn-QE. (C) Localization results from Syn-TOF. The numbers of correctly localized PSMs at 1\% FLR are indicated. Localization results are sorted by the deltaDP scores, Ascore, phosphoRS probabilities or pSite scores in descending order. 
A

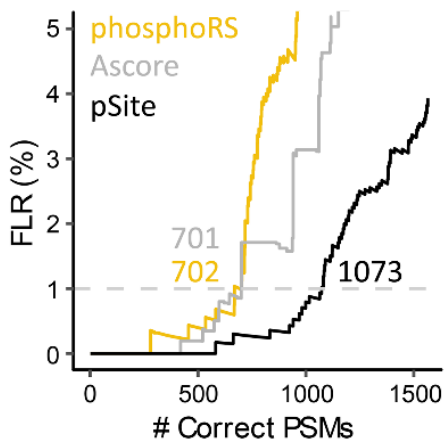

C Prosit

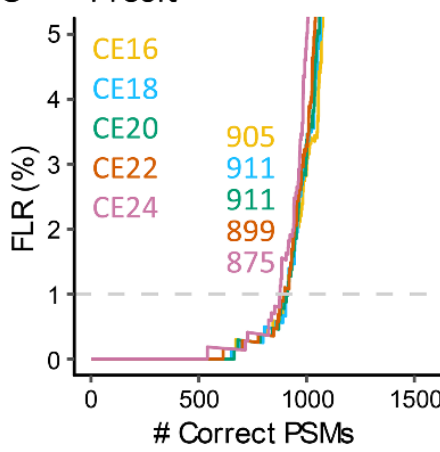

B
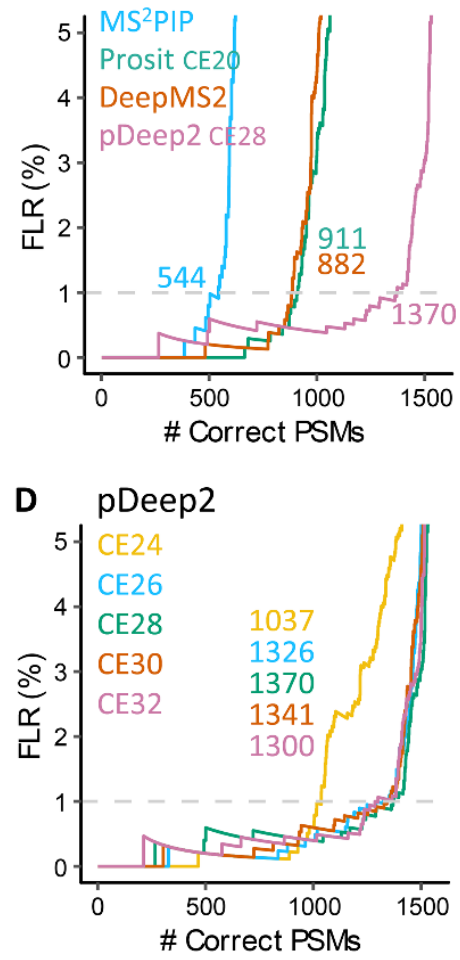

Figure S7. Phosphorylation site localization on Syn-QE of synthetic phosphopeptides using different methods. (A) Plots of PSM level FLR against the number of correctly localized PSMs by Ascore, pSite and phosphoRS, as well as (B) spectral matching using MS/MS spectra predicted by MS 2 PIP, Prosit, DeepMS2, and pDeep2. (C) Results of spectral matching using MS/MS spectra predicted by Prosit with different collision energy (CE) values. (D) Results of spectral matching using MS/MS spectra predicted by pDeep2 with different CE values. (E) Results of phosphoRS with the neutral loss (NL) option enabled or not. The numbers of correctly localized PSMs at $1 \%$ FLR are indicated. Localization results of PSMs are sorted by the deltaDP scores, Ascore, pSite scores or phosphoRS probabilities in descending order. No phosphoric acid neutral loss nor budding strategy was adopted for the in silico spectra generation. 
A
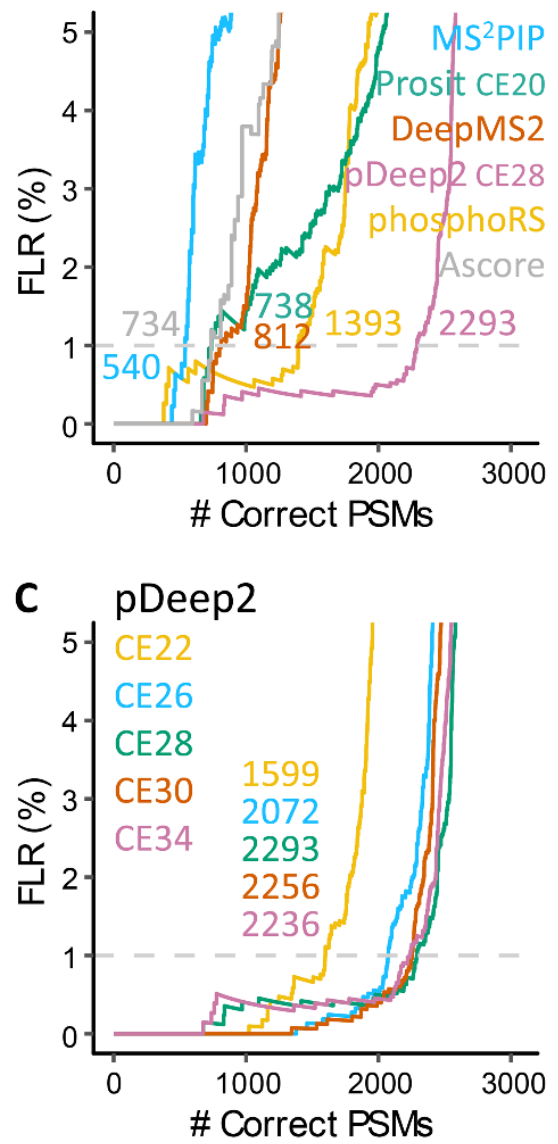

B

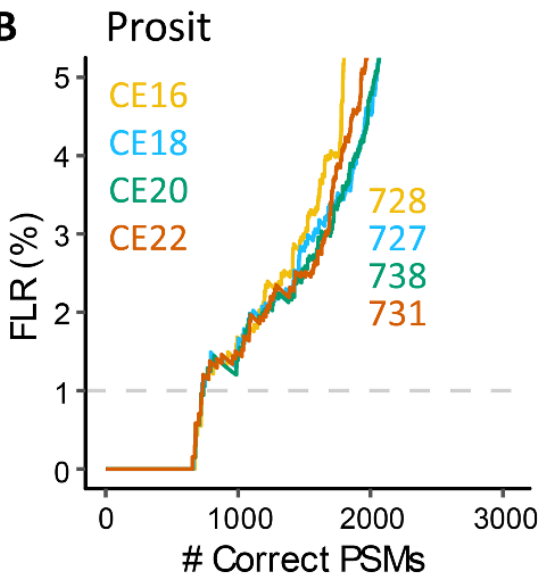

D phosphoRS

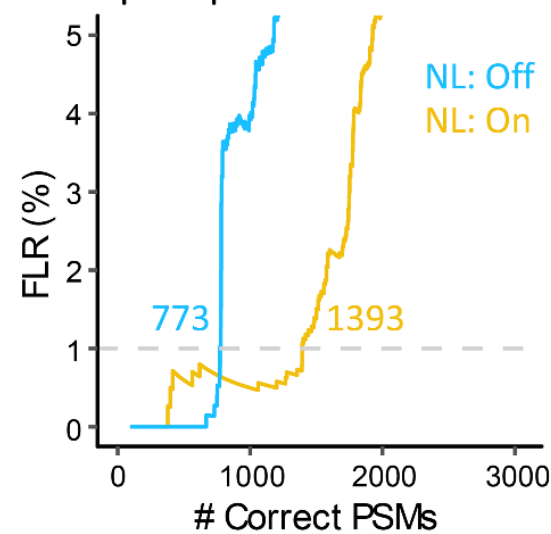

Figure S8. Phosphorylation site localization on Syn-TOF of synthetic phosphopeptides using different methods. (A) Plots of PSM level FLR against the number of correctly localized PSMs by Ascore and phosphoRS, as well as spectral matching using MS/MS spectra predicted by MS ${ }^{2}$ PIP, Prosit, DeepMS2, and pDeep2. (B) Results of spectral matching using MS/MS spectra predicted by Prosit with different collision energy (CE) values. (C) Results of spectral matching using MS/MS spectra predicted by pDeep2 with different CE values. (D) Results of phosphoRS with the neutral loss (NL) option enabled or not. The numbers of correctly localized PSMs at 1\% FLR are indicated. Localization results of PSMs are sorted by the deltaDP scores, Ascore, or phosphoRS probabilities in descending order. No phosphoric acid neutral loss nor budding strategy was adopted for the in silico spectra generation. 

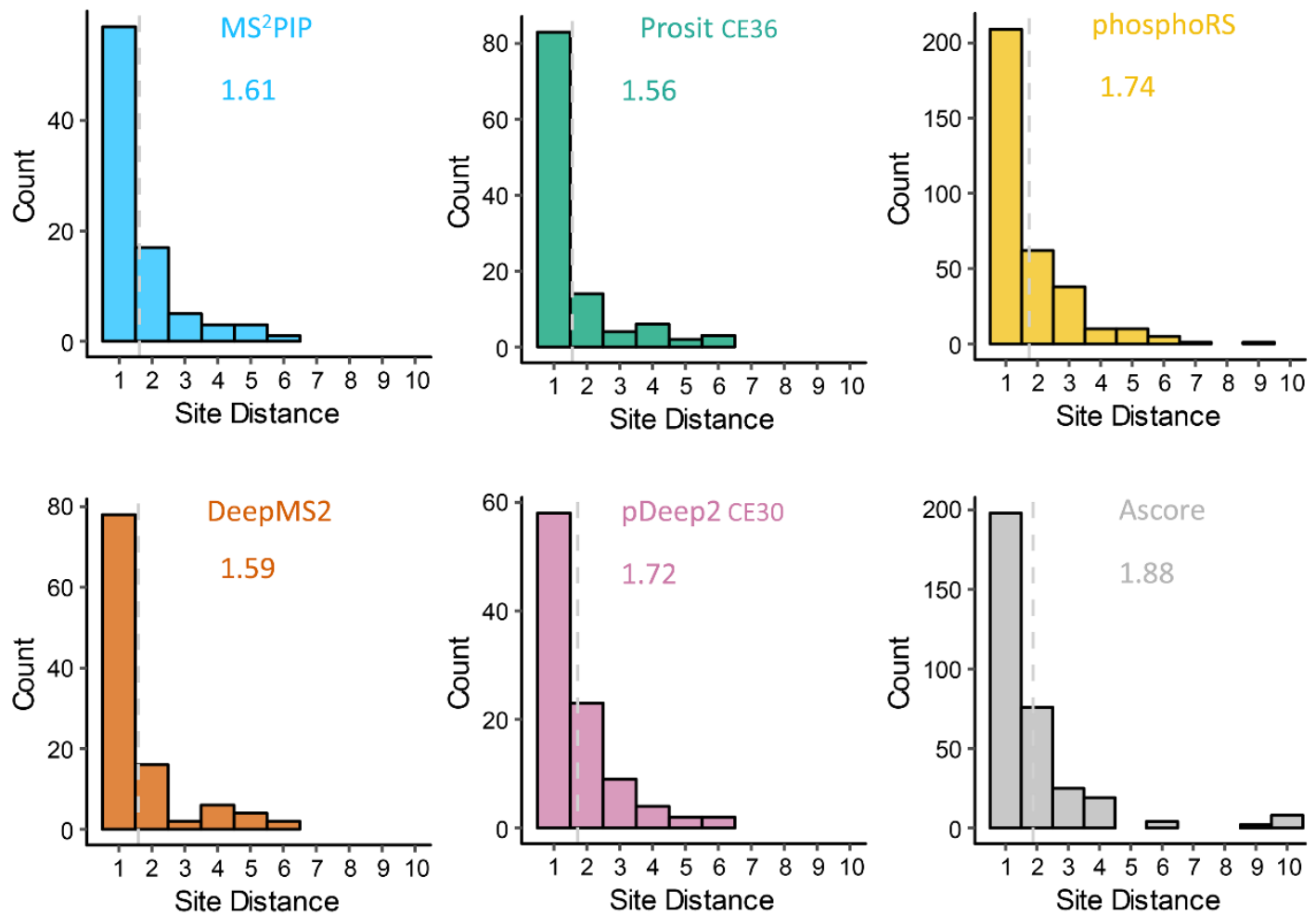

Figure S9. Distributions of distance in amino acid numbers between the mislocated phosphorylation sites (including ambitious localizations) and the correct sites in SynOF using different methods. Mean distances are indicated. 


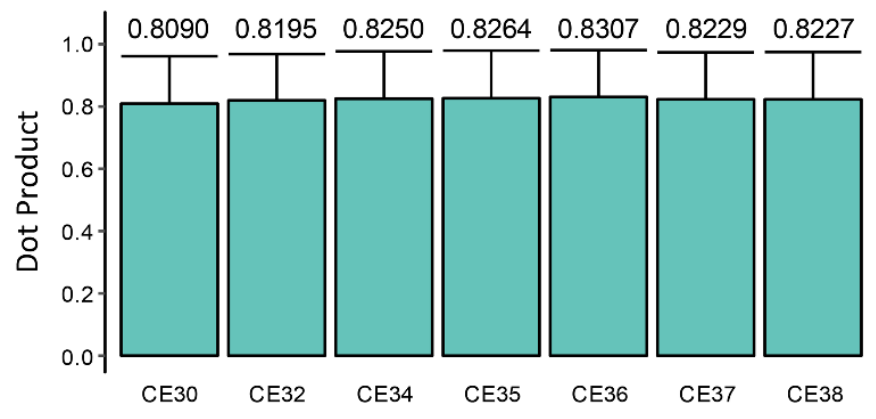

Figure S10. Dot product (DP) between the experimental intensities of $b / y$ ions and predicted intensities by pDeep2 with different collision energy (CE) values. Mean DPs were indicated. Error bars represent standard deviation. 


\section{A}

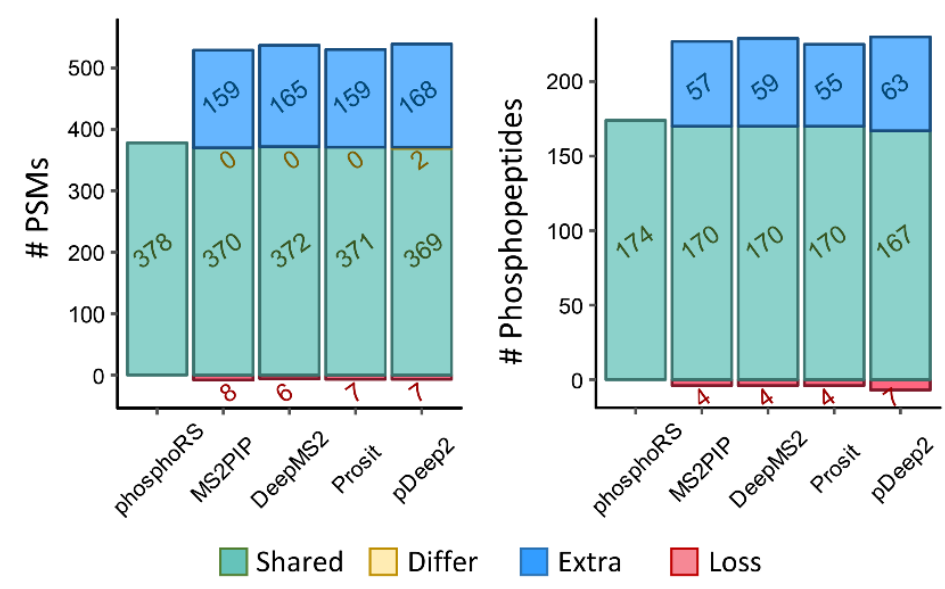

B

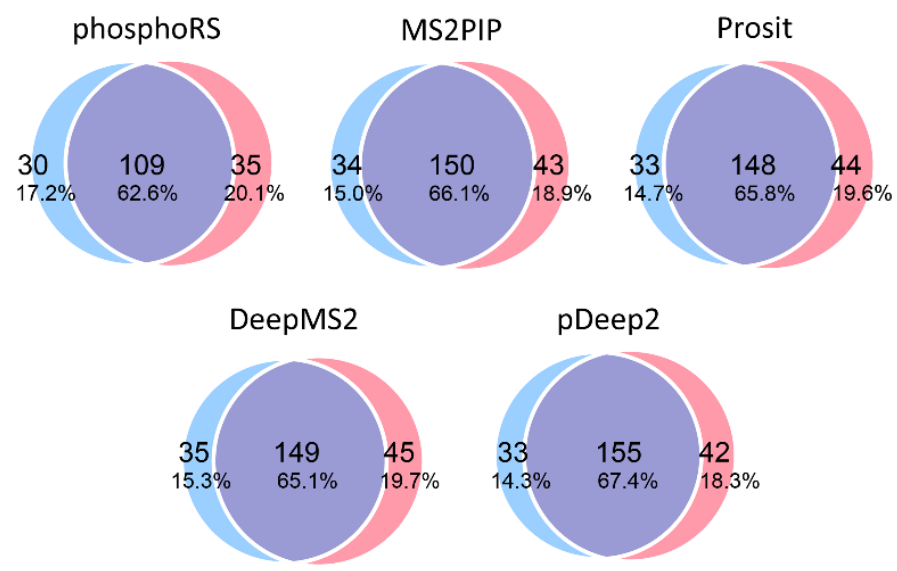

Figure S11. Phosphorylation site localization on doubly phosphorylated peptides from a data set of HeLa cells using different methods. (A) Numbers of PSMs and phosphopeptides localized by phosphoRS, alone or combined with spectral matching (DP-phosphoRS) using MS/MS spectra predicted by MS ${ }^{2}$ PIP, Prosit, DeepMS2, and pDeep2. Shared: PSMs with the same localization result by DP-phosphoRS as that by phosphoRS alone; phosphopeptides reported by both phosphoRS alone and DPphosphoRS. Differ: PSMs with different localization result by DP-phosphoRS from that by phosphoRS alone. Extra: spectra localized by DP-phosphoRS but not localized by phosphoRS alone; phosphopeptides only reported by DP-phosphoRS. Loss: spectra not localized by DP-phosphoRS but localized by phosphoRS alone; phosphopeptides only reported by phosphoRS alone. (B) Overlap of localized phosphopeptides between two technical replicates. 